\title{
Ist-Stand der Fachliteratur zu Effekten des therapeutischen Kletterns auf die psychische Gesundheit - Fazit: viel zu tun
}

\author{
Anika Frühauf $\mathbb{D} \cdot$ Kathrin Sevecke $\cdot$ Martin Kopp
}

Eingegangen: 4. April 2018 / Angenommen: 2. August 2018 / Online publiziert: 20. August 2018

(c) Der/die Autor(en) 2018

\begin{abstract}
Zusammenfassung
Hintergrund Therapeutisches Klettern wird sowohl zur Verbesserung physiologischer als auch psychologischer Zustandsbilder im Rehabilitationsbereich angewendet. Nachdem Überblicksarbeiten zu physiologischen Effekten der Klettertherapie durchgeführt wurden, soll diese Arbeit einen Überblick über die wissenschaftliche Evidenz psychologischer Effekte der Klettertherapie liefern.

Methodik Die Literaturrecherche erfolgte in englischer und deutscher Sprache in den wissenschaftlichen Datenbanken PubMed, Scholar und Science Direct (bis Dezember 2017). Als Einschlusskriterium wurde die empirische Erhebung psychologischer Effekte in der Klettertherapie vorausgesetzt.

Ergebnisse Insgesamt wurden sieben Artikel in die Arbeit eingeschlossen. Die Probandengruppen waren erwachsene Personen mit psychischen und Verhaltensstörungen a) im stationären $(n=3)$ und b) ambulanten Setting $(n=1)$, c) Kinder mit motorischen Störungen, bzw. Defiziten $(n=2)$ und d) gesunde Erwachsene $(n=1)$. Alle Artikel wiesen teils erhebliche methodische Limitationen auf. Drei der insgesamt sechs Artikel erhoben die Selbstwirksamkeit und zeigten Verbesserungen gegenüber der Kontrollgruppe. Unterschiede zwischen einem Kletter- und Fitnesstraining zeigten sich bei einer gesunden Probandengruppe nicht. Positive Veränderungen in der affektiven Befindlichkeit zeigten sich bei Personen
\end{abstract}

\footnotetext{
A. Frühauf $(\bowtie) \cdot$ M. Kopp

Institut für Sportwissenschaft, Universität Innsbruck, Fürstenweg 185, 6020 Innsbruck, Österreich anika.fruehauf@uibk.ac.at

K. Sevecke

Universitätsklinik für Psychiatrie, Psychotherapie und Psychosomatik im Kindes- und Jugendalter, Innsbruck, Österreich
}

mit schwerer depressiver Störung nach einer Klettereinheit im Vergleich zu einer Entspannungseinheit. Diskussion Die wissenschaftliche Evidenz für die Effektivität einer Klettertherapie auf psychologische Komponenten ist sehr gering. Möglicherweise führt Klettertherapie zu einer erhöhten Selbstwirksamkeit. Eine allgemeingültige Aussage kann jedoch auf Basis der aktuellen Datenlage nicht getroffen werden, da die Studien unterschiedliche Altersgruppen (Kinder und Erwachsene) sowie erhebliche Limitationen aufwiesen. Es bedarf kontrollierter Interventionsstudien, insbesondere Studiendesigns, die Klettertherapie mit aerober Bewegungstherapie bei Personen mit psychischen und Verhaltensstörungen vergleichen.

Schlüsselwörter Klettern · Therapieform · Psychische und Verhaltensstörungen - Psychologische Effekte, Kinder

\section{Current state of the scientific literature on effects of therapeutic climbing on mental health - conclusion: a lot to do}

\section{Summary \\ Background Therapeutic Climbing is applied in phys- iological as well as in psychological rehabilitation pro- grams. Systematic reviews have been conducted, eval- uating the physiological effects of therapeutic climb- ing. The aim of the following article is a review on existing literature about the psychological effects of climbing therapy. \\ Methods We searched the databases of PubMed, Scholar and ScienceDirect up to December 2017 in German and English language. Trials were included if they assessed psychological variables in climbing interventions. \\ Results Seven articles were included in the follow- ing review. Effectiveness of climbing therapy was}


studied in a) adult inpatients with mental disorders $(n=3)$, b) adult outpatients with mental disorders $(n=1)$, c) children with motoric deficits and disabilities $(n=2)$, d) healthy adults $(n=1)$. All trials had major methodological limitations. Control groups if present, were defined differently. Three trials assessed self-efficacy and revealed enhanced self-efficacy after the climbing intervention. No psychological differences were seen between aerobic exercise and climbing therapy in healthy adults. Acute positive affective changes were seen in in-patients with major depressive disorders after a climbing session compared to a relaxation session.

Conclusion The effectiveness of climbing therapy on psychological outcomes remains unclear. Limited evidence with varying age groups (children and adults) and a high risk of bias shows enhanced self-efficacy through climbing therapy. There is a need for further studies in children and adults on psychological outcomes through climbing therapy, especially in comparison with aerobic activity interventions.

Keywords Climbing · Therapeutic interventions · Psychological effects $\cdot$ Psychiatric patients $\cdot$ Children

\section{Einleitung}

Regelmäßige körperliche Aktivität senkt das Mortalitätsrisiko und hat Einfluss auf die kognitiven Fähigkeiten und die psychische Gesundheit [1]. Bewegungstherapeutische Maßnahmen sind kostengünstig, leicht durchführbar und haben im Gegensatz zu anderen Behandlungsstrategien wie Pharmakotherapie kaum Nebenwirkungen [2]. Evidenzbasierte Untersuchungen konnten zeigen, dass körperliche Aktivität förderlich bei der Behandlung von psychischen und Verhaltensstörungen ist [3], insbesondere bei Personen mit depressiven Störungen [4, 5] und Angststörungen [6]. Die häufigsten Interventionsformen sind dabei aerobe Bewegungsinterventionen wie Gehen oder Fahrradfahren auf einem Ergometer [7].

Der Klettersport gilt als beliebter Freizeit- und Leistungssport, welcher immer mehr Anhänger findet $[8,9]$. Dies zeigt sich unter anderem auch in der Aufnahme der Kletterdisziplinen (Bouldern'; ${ }^{1}$ Lead ${ }^{2}$; Speedklettern $^{3}$ ) in das olympische Sportprogramm ab dem Jahr 2020 [10]. Auch in der Wissenschaft gibt es zahlreiche Artikel, die sich dem Klettersport widmen und beispielsweise die Kraftverteilung, Überlastungsschäden und Verletzungen im Klettersport untersuchten [11-14]. In Bezug auf therapeutisches Klettern gibt es jedoch kaum wissenschaftliche Untersuchungen. „Therapeutisches Klettern - kaum erforscht und

\footnotetext{
1 Klettern auf Absprunghöhe.

2 Vorstiegsklettern, Seilsicherung von unten.

3 Gleichbleibende Route, Klettern auf Geschwindigkeit mit Seilsicherung von oben.
}

dennoch zunehmend eingesetzt“, so lautete eine Übersichtsarbeit aus dem Jahr 2011 von Grzybowski und Eils [15]. Sie zeigten auf, welche physiologischen Wirkungen Klettertherapie bei orthopädischen und neurologischen Beschwerden sowie bei psychomotorischen und angeborenen/chronischen Erkrankungen haben kann. Allerdings wiesen die eingeschlossenen Studien hohe methodische Limitationen auf. Die Autoren kamen zu dem Schluss, dass es nur mangelnde Nachweise für die physiologische Effektivität eines therapeutischen Kletterprogrammes gibt. In einem weiteren Review aus dem Jahr 2011 wurden ebenfalls keine zufriedenstellenden Nachweise für die Effektivität einer therapeutischen Kletterintervention in der Prävention oder in der Behandlung von körperlichen Gesundheitsproblemen gefunden [16].

\section{Doch was ist mit den psychologischen Aspekten?}

Dass der Klettersport therapeutisches Potential hat, wurde schon vor über 120 Jahren vermerkt [17]. Weber [17] empfahl den Klettersport insbesondere Personen, die sich mental stark beanspruchten und hauptsächlich Büroarbeit verrichten und eventuell dadurch häufige Symptome wie Übergewicht, Schlafprobleme, Gereiztheit und Deprimiertheit zeigten. Bei nicht vorliegenden organischen Erkrankungen empfahl er einen Aufenthalt in den Bergen, um Gesundheit und Lebensenergie zurückzugewinnen. Somit scheint die Anwendung des Klettersports im psychologischen Bereich weiter zurückzugehen als die physiologische Anwendung. Als erste therapeutische Kletterinterventionen nannte Lazik [18] in den 80er Jahren das Klettern als Therapieform im Strafvollzug. Hierbei wurden vor allem die psychosozialen Aspekte des Kletterns als Partnersport gefördert [18]. Beim Klettern werden physiologische, sowie psychologische und kognitive Ansprüche gestellt. Neben muskulärem Zuwachs und der Verbesserung koordinativer Fähigkeiten könnte durch die Überwindung physischer und psychischer Grenzen und Ängste eine realistischere Selbsteinschätzung entwickelt sowie das Selbstvertrauen gesteigert werden. Auch soziale Aspekte, wie Vertrauen in den Sicherungspartner, Verantwortungsbewusstsein und Kooperation, könnten durch Klettern geschult werden [19]. Leichtfried hebt den positiven Anforderungscharakter des Kletterns hervor, welcher vor allem im Zusammenhang mit psychischen und Verhaltensstörungen förderlich erscheint [19]. Beim Klettern wird Kreativität und Spontanität gefördert. Es erfordert Lösungsstrategien zum Bewältigen der Routen, das Einhalten von Regeln, sowie Verantwortungsbewusstsein und Kooperation kombiniert mit Aufmerksamkeit und innerer Wachsamkeit [19]. Dies erscheint besonders förderlich bei Patienten mit einer Aufmerksamkeitsdefizit-/Hyperaktivitätsstörung (ADHS) [20]. Veser [20] beschreibt dies bei einer therapeutischen Klettergruppe von Kindern mit ADHS folgendermaßen: „Vielmehr fördert die Klettertherapie 
auch grundlegende kognitive und sozio-emotionale Fähigkeiten wie Konzentration und Merkfähigkeit oder das Vertrauen in Therapeuten und Sicherungspartner. Das Kind lernt auch, Handlungsabläufe zu planen und einzuhalten - und es erhält eine unmittelbare und konkrete Rückmeldung durch den Kletterablauf selbst." Trotz dieser positiven Vermerke erscheint die empirische Datenlage unzureichend und vornehmlich auf physiologische Effekte der Klettertherapie beschränkt [15, 16, 21]. Im Folgenden wird ein Überblick über die aktuelle Datenlage der Klettertherapie als Anwendungsform im psychologischen Bereich gegeben.

\section{Methodik}

Die Literaturrecherche erfolgte von Juli bis Dezember 2017. In erster Linie wurden empirische Studien zu den psychologischen Effekten durch Klettertherapie gesucht. Gesucht wurde in den Datenbanken PubMed, ScienceDirect, Wiley Online Library, Scholar und über die Schlagwörter „therapeutic climbing“, „climbing“ „therapy“, „bouldering“, „health“, ,indoor climbing“, „rock climbing“ in englischer und deutscher Sprache. Des Weiteren wurde auf Bücher zurückgegriffen, die das Thema therapeutisches Klettern behandeln. Bei allen Artikeln wurden die Literaturverzeichnisse berücksichtigt und gegebenenfalls in die Datenbank miteinbezogen. Aufgrund der mangelnden Datenlage wurden auch Fallbeispiele begutachtet.

\section{Ergebnisse}

\section{Empirische Studien}

Es wurden sieben empirische Studien gefunden, die psychologische Effekte der Klettertherapie untersuchten (Tab. 1). Alle Studien wiesen teils erhebliche methodische Limitationen auf.

Vier Studien untersuchten die Auswirkungen der Klettertherapie bei erwachsenen Patienten mit psychischen und Verhaltensstörungen [22-24]. Von diesen wurden vier Studien in einem naturalistischem Setting an stationären Patienten [22, 23, 25] durchgeführt und eine Studie an ambulanten Patienten [24]. Die Studien variierten in ihrer Interventionsart (Hochseilgarten, Seilklettern, Bouldern), Interventionsdauer (einmalige Intervention, mehrwöchige Intervention) sowie in der Definition der Kontrollgruppe (keine Kontrollgruppe, Patienten ohne Kletterintervention, Entspannungstechnik, Warteliste). Der Hochseilgarten, wie er in der Studie von Wolf und Mehl [23] beschrieben wurde, ist ein konstruiertes Gerüst, das $12 \mathrm{~m}$ hohe Baumstämme durch Drahtseile verbindet. Zwischen den Baumstämmen gibt es statische und schwingende Elemente aus Seilen und Holzbalken mit verschiedenen Übungsmöglichkeiten. Die partizipierenden Personen werden während der Benutzung des Hochseilgartens durch Seilsicherung an einem Klet- ter- und Brustgurt vor Stürzen gesichert. Die Übungsabfolge wurde in der angeführten Untersuchung mit allen Patienten individuell erarbeitet. In der Studie von Kleinstäuber et al. [25] wurde eine einmalige Seilklettereinheit mit einer Entspannungseinheit verglichen. Die Entspannungseinheit beinhaltete eine angeleitete Entspannung (Progressive Muskelrelaxation nach Jacobson) mit anschließender Reflexion. Die Klettereinheit umfasste ein spielerisches Aufwärmen an der Kletterwand ohne Seil (im Absprungbereich), gefolgt von einer Seilkletterintervention mit anschließender Reflexion. Dabei wurden die jeweiligen Patienten von geschultem Personal gesichert. Kleinstäuber et al. [25] zeigten positive Veränderungen in der affektiven Befindlichkeit nach einer einmaligen Klettereinheit im Vergleich zu einer Entspannungseinheit. In der Studie von Luttenberger et al. [24] wurde nur Bouldern - das Klettern auf Absprunghöhe - praktiziert. Daher wurden keine Materialschulungen in der Intervention eingebaut. Zusätzlich zu dem kletterspezifischen Training, wurde in jeder Einheit ein verhaltensrelevantes Thema (z. B. „Angst und Vertrauen“) erarbeitet und reflektiert. Sowohl die Daten von Wolf und Mehl [23] als auch von Luttenberger et al. [24] zeigten eine verbesserte Selbstwirksamkeit und Veränderungen in der Zustandsangst bei der Interventionsgruppe im Vergleich zur Kontrollgruppe.

Zwei Studien wurden bei Kindern (8-12 Jahre) durchgeführt, die an physiologischen Beeinträchtigungen litten (Zerebralparese ${ }^{4}$, bzw. motorischen Defiziten $^{5}$ ) [26, 27]. Schram Christensen et al. [27] führten eine kombinierte Kletterintervention (Bouldern und Seilklettern) durch. Mazzoni et al. [26] beschrieben eine Seilkletterintervention. Bei beiden Studien lag der Fokus auf physiologischen Veränderungen. Durch eine zusätzliche Erhebung psychologischer Variablen zeigte sich bei Mazzoni et al. [26] eine verbesserte Selbstwirksamkeit in der Interventionsgruppe im Vergleich zur Kontrollgruppe. Schram Christensen et al. [27] fanden zwischen ihrer Interventionsgruppe bei Kindern mit Zerebralparese und Kindern ohne Beeinträchtigungen keine Unterschiede in psychologischen Variablen.

Eine Studie untersuchte die psychischen Auswirkungen einer Kletterintervention im Vergleich zu einer Fitnessintervention bei gesunden Probanden [28]. Die Kletterintervention umfasste ein Klettertraining mit Seilsicherung. Neben dem kletterspezifischen Training wurden auch der Umgang und die Anwendung der Sicherheitsausrüstung geschult. Die Zuteilung der Gruppen erfolgte selbstgewählt. Es ergab sich kein signifikanter Unterschied zwischen den Gruppen hinsichtlich des Angstzustandes. Sowohl in der

\footnotetext{
4 = zumeist angeborene, frühkindliche Bewegungsstörung.

5 eingeschlossen wurden Kinder die aufgrund ihrer motorischen Beeinträchtigungen bereits therapeutische Hilfe in Anspruch nahmen, sowie jene die weniger als $15 \%$ bei dem motorischen Test, Movement Assessment Battery for Children` erreichen.
} 


\section{übersicht}

\begin{tabular}{|c|c|c|c|c|c|c|c|c|}
\hline & 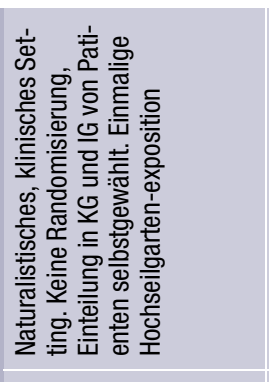 & 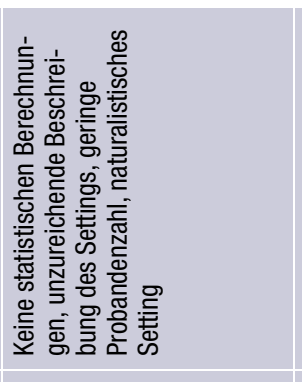 & 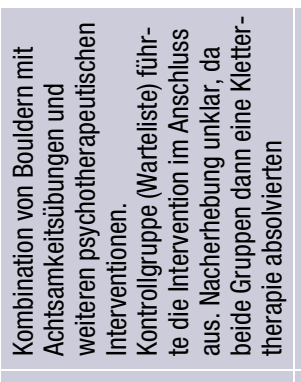 & 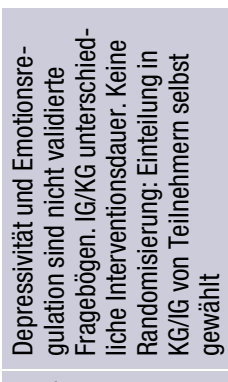 & 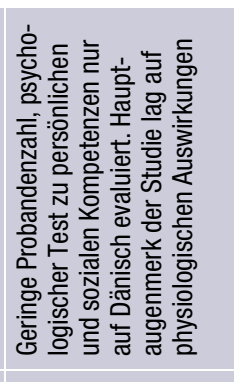 & 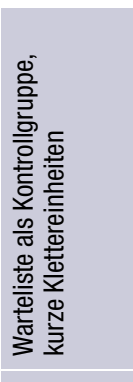 & 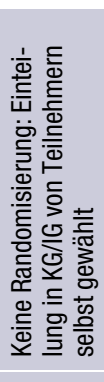 & 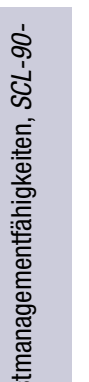 \\
\hline & 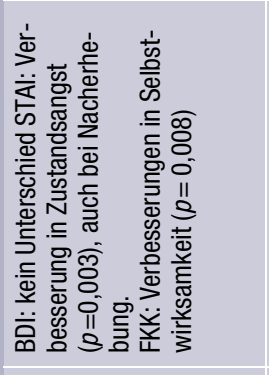 & 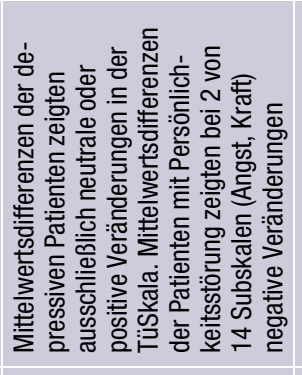 & 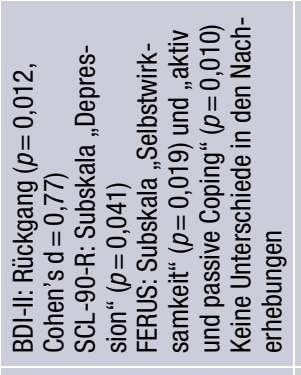 & 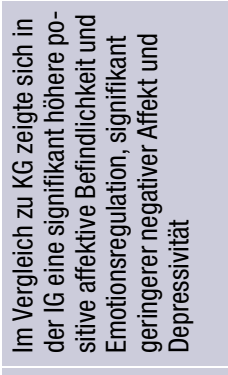 & 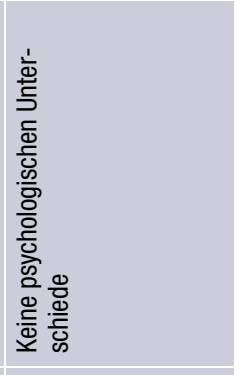 & 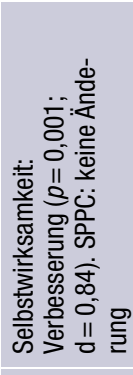 & 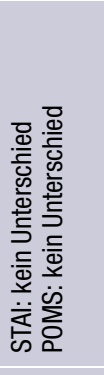 & 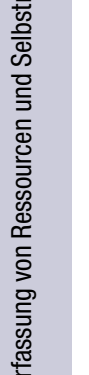 \\
\hline & & 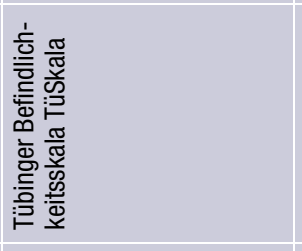 & 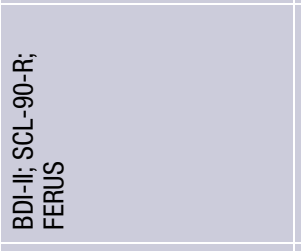 & 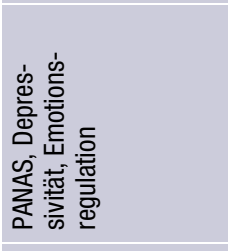 & 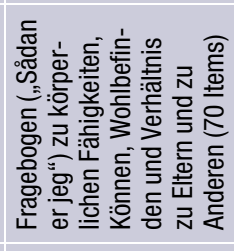 & 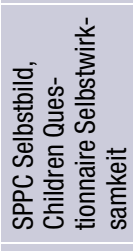 & $\begin{array}{l}\frac{\infty}{2} \\
\frac{2}{0} \\
\frac{1}{5}\end{array}$ & 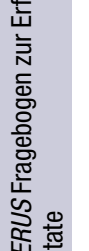 \\
\hline & 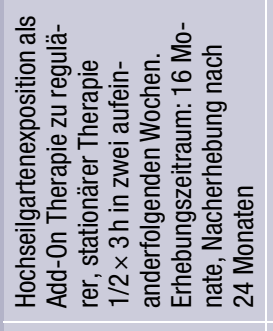 & 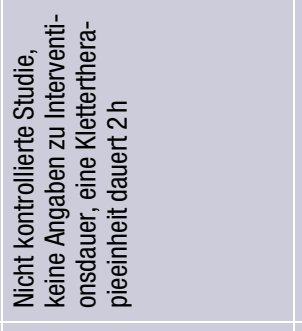 & 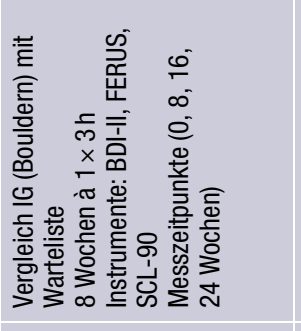 & 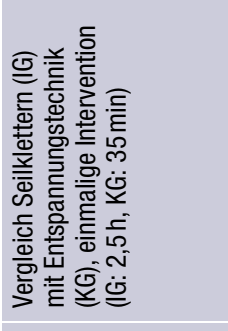 & 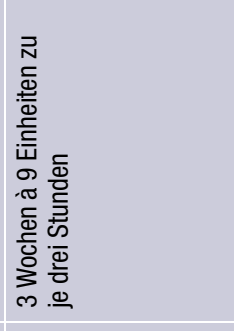 & 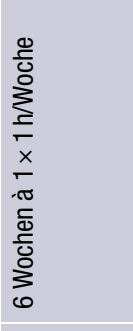 & 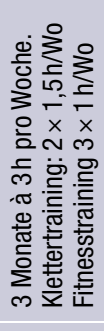 & 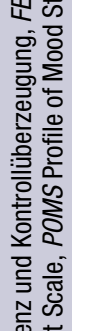 \\
\hline & 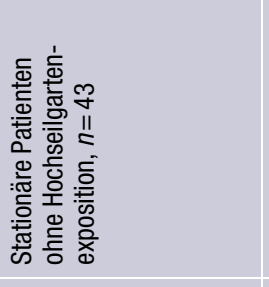 & 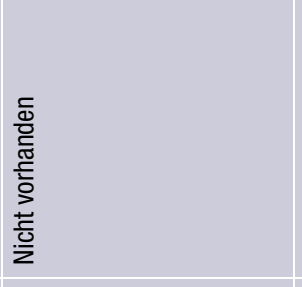 & 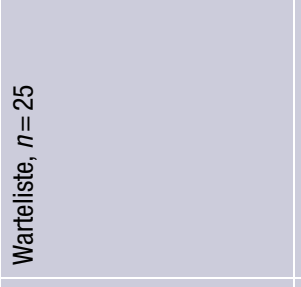 & 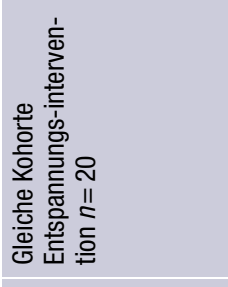 & 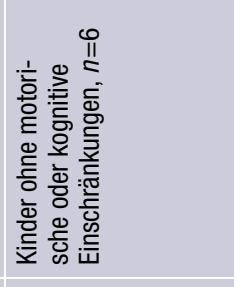 & 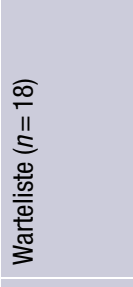 & 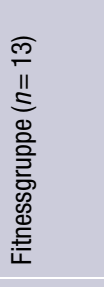 & \\
\hline & 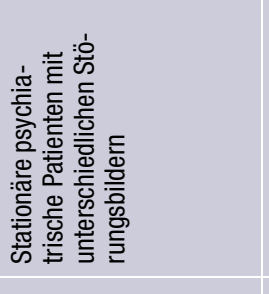 & 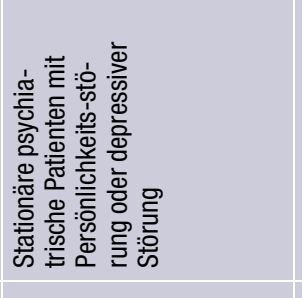 & 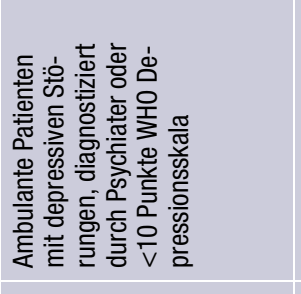 & 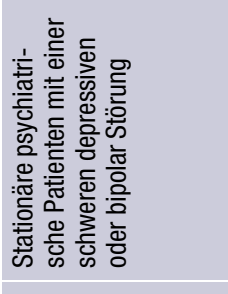 & 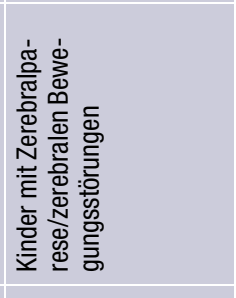 & 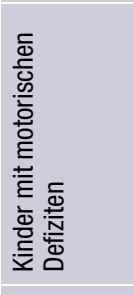 & 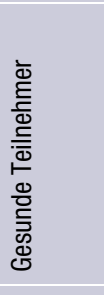 & 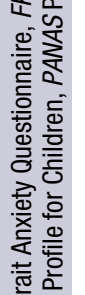 \\
\hline 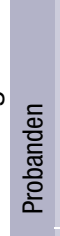 & 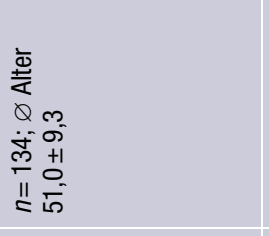 & 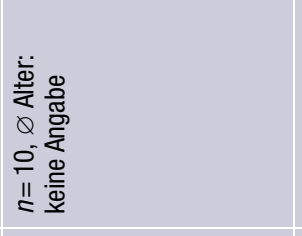 & 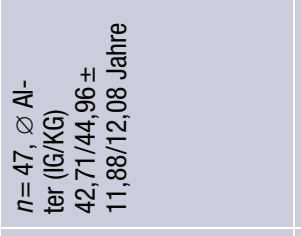 & 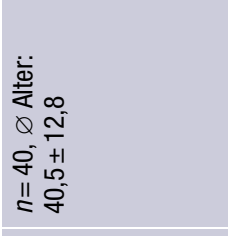 & 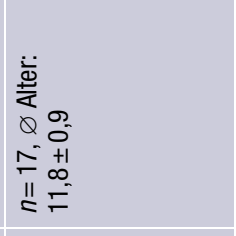 & 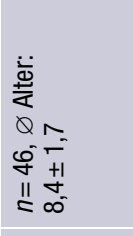 & 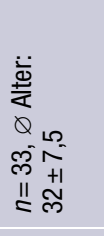 & 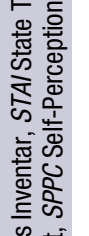 \\
\hline & 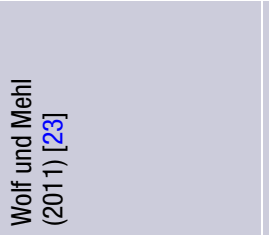 & 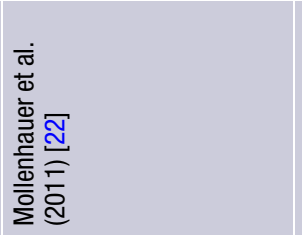 & 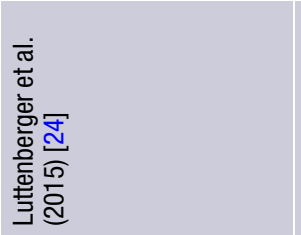 & 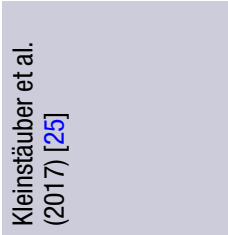 & 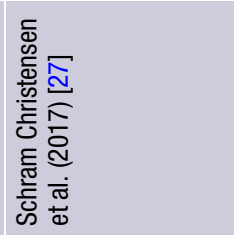 & 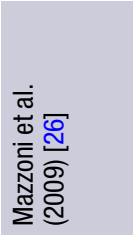 & 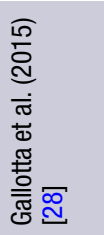 & 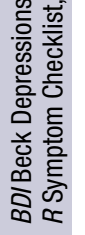 \\
\hline
\end{tabular}


Fitness- als auch in der Klettergruppe wurden nach einer einzelnen Einheit reduzierte Ausprägungen der Zustandsangst festgestellt.

\section{Fallbeispiele}

Aufgrund der geringen Studienanzahl soll der Bericht von Fallbeispielen einen erweiterten Einblick in die Klettertherapie geben.

Wallner beschrieb Einzelfallanalysen von acht jungen Erwachsenen $(\varnothing 24,4 \pm 4,1$ Jahre), welche sie im Schuljahr 2009/2010 klettertherapeutisch betreute. Sechs Personen kletterten dabei im Gruppensetting einmal pro Woche (Dauer nicht angeführt), zwei Personen kletterten aufgrund der erhöhten Ablenkbarkeit in Einzelsettings (Dauer und Umfang nicht angeführt). Die Probanden waren mit leichten Lernschwächen bis hin zu mittelgradigen Intelligenzminderungen diagnostiziert. Manche Teilnehmer wiesen zusätzliche psychische und Verhaltensstörungen wie Somatisierungsstörung, ADHS oder Essstörung auf. Als wichtigste beobachtete Handlungen beschrieb Wallner Veränderungen im kognitiven, sozialen, emotionalen und motorischen Bereich [29]. Im kognitiven Bereich beobachtete sie eine Verbesserung der Konzentration, eine geringere Ablenkbarkeit und eine realistischere Zielsetzung bei den TeilnehmerInnen. Im sozialen Bereich beobachtete sie eine erhöhte soziale Kompetenz, eine Stärkung der Klarheit in der Kommunikation und Freude bei der Verantwortungsübernahme. Im emotionalen Bereich berichtete Wallner unter anderem von einer beobachteten Verbesserung im Selbstbewusstsein und einer erhöhten Frustrationstoleranz.

In dem Buch von Kowald und Zajetz „Therapeutisches Klettern. Anwendungsfelder in Psychotherapie und Pädagogik." werden weitere Fallberichte beschrieben [30]. Susanne Wallner, Claudia Kern und Stefan Jenuwein berichteten an ausgewählten Beispielen über ihre Erfahrungen mit der Klettertherapie. Es wurden Fallbeispiele für Erwachsene mit unterschiedlichen Störungsbildern (Multiple Sklerose $[n=1]$, schwere depressive Störung [ $n=1]$, Höhenangst $[n=1])$, sowie für Kinder und Jugendliche mit unterschiedlichen Störungen (Kind mit verbalem und aggressivem Verhalten [ $n=1]$, Jugendlicher mit desorganisiertem Bindungsverhalten und ADHS $[n=1]$ ) beschrieben. Zusammenfassend berichteten die Autoren von einer Steigerung im Bereich Selbstwert und einer Verbesserung der sozialen Fertigkeiten der Personen durch die Klettertherapie.

\section{Diskussion}

Sieben Artikel wurden in die Überblicksarbeit miteingeschlossen, wobei Personengruppen, Kontrollgruppen, Altersgruppen, Erhebungsparameter, Interventionsart und -dauer, stark variierten. Von den insgesamt sieben eingeschlossenen Artikeln erhoben drei Artikel die Selbstwirksamkeit und fanden signifikante Veränderungen bei der Versuchsgruppe, konnten diese Verbesserungen jedoch nur teilweise mit einer Kontrollgruppe kontrollieren [23, 24, 26]. Bezogen auf die Altersgruppe zeigte sich dies sowohl bei Erwachsenen als auch bei Kindern. Bei psychiatrischen Patienten zeigte sich eine Verbesserung in der Zustandsangst durch eine Hochseilgartenexposition im Vergleich zur Kontrollgruppe [23]. Ähnliche Verbesserungen in der Zustandsangst zeigten sich auch bei gesunden Probanden, allerdings waren diese auch durch eine Fitnesstrainingsintervention zu finden [28]. Vermutlich wurden diese Veränderungen daher nicht durch die Klettertherapie, sondern durch die körperliche Aktivität ausgelöst. Akut ließen sich signifikante Verbesserungen der affektiven Befindlichkeit durch eine Kletterintervention bei Patienten mit schweren depressiven und bipolaren Störungen im Vergleich zu einer Entspannungsintervention nachweisen [25].

Bei ambulanten Patienten mit depressiver Störung wurde durch eine achtwöchige Klettertherapie ein Rückgang im Beck Depressions Inventar II erreicht, welcher sich signifikant von Personen der Warteliste unterschied [24]. Dies ist unseres Wissens die erste randomisiert-kontrollierte Studie zu den Effekten der Klettertherapie bei einer Probandengruppe mit psychischen und Verhaltensstörungen, wobei die Diagnose nicht einheitlich erhoben wurde (entweder durch einen Arzt oder durch einen Fragebogen). Als Interventionsart wurde Bouldern gewählt. Die Intervention fand einmal wöchentlich über drei Stunden statt. Allerdings ist aus der Beschreibung der Interventionseinheiten ersichtlich, dass auch ergänzende Achtsamkeitsübungen und psychotherapeutische Verfahren eingesetzt wurden. Somit können die Effekte nicht einzig auf die klettertherapeutischen Maßnahmen zurückgeführt werden. Als Kontrollgruppe wurde eine Warteliste gewählt, welche keine therapeutische Intervention erfuhr. Eine Warteliste als Kontrollgruppe zu erheben birgt die Gefahr, den Effekt der Intervention zu überschätzen [31]. Zudem kann durch die Studie von Luttenberger et al. [31] keine Aussage über die Effektivität einer Klettertherapie verglichen mit einer anderen Bewegungsintervention bzw. einer Kontaktgruppe, welche dieselbe Art der therapeutischen Intervention erhält, abgeleitet werden. Ein Vergleich zwischen einer Bewegungsintervention und einer Kletterintervention wurde lediglich bei einer gesunden Probandengruppe durchgeführt. Nach einer dreimonatigen Interventionszeit wurden keine Gruppenunterschiede zwischen den Sportarten erkannt [28]. Allerdings erfolgte die Gruppenzuteilung nicht randomisiert, sondern von den Probanden selbst gewählt. Zwei Studien hatten das primäre Ziel physiologische Unterschiede durch die Kletterinterventionen festzustellen, da die Probandengruppe aus Kindern mit motorischen Defiziten, bzw. Störungen bestand. Zusätzlich zu den physiologischen Parametern wurden Veränderungen in der psychischen 
Gesundheit erhoben. Schram Christensen et al. [27] konnten keine Veränderungen in Bezug auf die psychische Gesundheit berichten. Allerdings wurde der verwendete Fragebogen, der persönliche und soziale Komponenten erfasste, in der Studie nicht näher beschrieben. Mazzoni et al. [26] fanden Verbesserungen im Bereich der Selbstwirksamkeit in einer kontrollierten Studie bei Kindern mit motorischen Defiziten im Vergleich zu einer Wartelistekontrollgruppe. Unterschiede im Selbstbild konnte diese Studie nicht identifizieren. Die Klettereinheiten fanden einstündig über 6 Wochen je einmal wöchentlich statt. Die beschriebenen Fallanalysen geben einen Einblick, dass Klettertherapie bereits bei einer Vielzahl von Patienten mit psychischen und Verhaltensstörungen eingesetzt wurde und in Einzelfällen positive Auswirkungen hatte. Allerdings können diese Analysen nicht als wissenschaftliche Evidenz herangezogen werden, scheinen sie doch sehr von der Interaktion zwischen Instruktoren und Patienten beeinflusst. Leider lässt auch die Studie von Mollenhauer et al. [22] wenig wissenschaftliche Rückschlüsse auf die Effektivität zu, da keine Kontrollgruppe erhoben wurde und die Stichprobe sehr klein war. Eine allgemeingültige Aussage über die psychologischen Effekte der Klettertherapie zu treffen ist nicht möglich, da die wenigen Studien verschiedene Altersgruppen (sowohl Kinder als auch Erwachsene) untersuchten und zudem zahlreiche Limitationen aufwiesen. Zusammenfassend lässt sich sagen, dass Praktizierende scheinbar gute Erfahrungen mit der Klettertherapie machten, dass aber die wissenschaftliche Evidenz dieser Intervention noch nicht bestätigt ist [22, 29, 30]. Langfristig zeigten sich keine psychologischen Unterschiede zwischen einer Kletterintervention und einer aeroben Trainingsintervention (bei einer gesunden Probandengruppe) [28]. Positive Veränderungen in der affektiven Befindlichkeit ließen sich jedoch, im Vergleich zu einer Entspannungseinheit, nach einer Klettereinheit an Patienten mit schweren depressiven Störungen, nachweisen [25]. Daher sollten zukünftige kontrollierte Studien Untersuchungen bei Personen mit psychischen und Verhaltensstörungen die Auswirkungen von Klettertherapie im Vergleich $\mathrm{zu}$ anderen Interventionen anstreben. Zudem wäre es erforderlich, mögliche Unterschiede zugunsten der Klettertherapie im Vergleich zu aerober körperlicher Aktivität wie Gehen oder Fahrradfahren, die viel einfacher in der Durchführbarkeit sind und Effekte in der Behandlung von Patienten mit psychischen und Verhaltensstörungen gezeigt haben nachzuweisen, um diese neue Bewegungsintervention auch evidenzbasiert $\mathrm{zu}$ rechtfertigen [3, 4, 6]. Auch die Erwartungshaltung der Probanden sowie die entgegengebrachte Aufmerksamkeit durch die Studienteilnahme scheint einen wichtigen Einfluss auf die Studienergebnisse zu haben [32]. Daher empfiehlt es sich in zukünftigen Studien auch auf eine nicht körperlich aktive Kontrollgruppe zu kontrollieren - wobei Interventionsgruppen (z. B. sozialer
Kontakt, Entspannungstechnik, Psychotherapie) der Warteliste als Kontrollgruppe vorzuziehen sind [31]. Ein anzustrebendes Studiendesign wäre somit eine randomisierte-kontrollierte Studie mit den drei Interventionsgruppen Klettern, aerobe Bewegung und soziale Kontaktgruppe bei einer homogenen Patientengruppe mit psychischen und Verhaltensstörungen.

\section{Schlussfolgerung}

Es bleibt fraglich, ob Klettertherapie, insbesondere für Personen mit psychischen und Verhaltensstörungen, mehr positive psychologische Effekte hat als andere Formen körperlicher Aktivität. Klettern wird häufig in der Behandlung von Patienten mit psychischen und Verhaltensstörungen bereits empfohlen [29, 30], obwohl die wissenschaftliche Evidenz dieser - im Vergleich zu anderen Bewegungsinterventionen - personal- und materialaufwändigen Intervention, derzeit nicht gegeben ist. Wie schon von Grzybowski und Eils [15] erwähnt wurde, sollen die fehlenden wissenschaftlichen Nachweise nicht zu einer Ablehnung der Klettertherapie führen, sondern als Forschungsanreiz zur Durchführung kontrollierter Studien in verschiedenen Altersgruppen (Kinder- und Jugendliche, sowie Erwachsene) verstanden werden.

Funding Open access funding provided by University of Innsbruck and Medical University of Innsbruck.

Interessenkonflikt A. Frühauf, K. Sevecke und M. Kopp geben an, dass kein Interessenkonflikt besteht.

Open Access Dieser Artikel wird unter der Creative Commons Namensnennung 4.0 International Lizenz (http:// creativecommons.org/licenses/by/4.0/deed.de) veröffentlicht, welche die Nutzung, Vervielfältigung, Bearbeitung, Verbreitung und Wiedergabe in jeglichem Medium und Format erlaubt, sofern Sie den/die ursprünglichen Autor(en) und die Quelle ordnungsgemäß nennen, einen Link zur Creative Commons Lizenz beifügen und angeben, ob Änderungen vorgenommen wurden.

\section{Literatur}

1. Biddle SJH, Asare M. Physical activity and mental health in children and adolescents: a review of reviews. $\mathrm{Br} \mathrm{J}$ Sports Med. 2011;45(11):886-95. https://doi.org/10.1136/ bjsports-2011-090185.

2. Taylor AH, Faulkner G. Inaugural editorial. Ment Health Phys Act. 2008;1(1):1-8. https://doi.org/10.1016/j.mhpa. 2007.11.001.

3. Pedersen BK, Saltin B. Exercise as medicine - evidence for prescribing exercise as therapy in 26 different chronic diseases. Scand J Med Sci Sports. 2015;25(Suppl 3):1-72. https://doi.org/10.1111/sms.12581.

4. CooneyGM, Dwan K, GreigCA, etal.Exercisefor depression. Cochrane Database Syst Rev. 2013;9:CD4366. https://doi. org/10.1002/14651858.CD004366.pub6.

5. Ekkekakis P. Honey, I shrunk the pooled SMD!: Guide to critical appraisal of systematic reviews and meta-analyses using the Cochrane review on exercise for depression as 
example. Ment Health Phys Act. 2015;8:21-36. https://doi. org/10.1016/j.mhpa.2014.12.001.

6. Stubbs B, Vancampfort D, Rosenbaum S, et al. An examination of the anxiolytic effects of exercise for people with anxiety and stress-related disorders: A meta-analysis. Psychiatry Res. 2017;249:102-8. https://doi.org/10.1016/j. psychres.2016.12.020.

7. Stanton R, Reaburn P. Exercise and the treatment of depression: a review of the exercise program variables. J Sci Med Sport. 2014;17(2):177-82. https://doi.org/10.1016/j.jsams. 2013.03.010.

8. Campbell AD, Davis C, Paterson R, et al. Preparticipation Evaluation for Climbing Sports. Wilderness Environ Med. 2015;26(4):40-6. https://doi.org/10.1016/j.wem. 2015.09.014.

9. Schweizer A. Sport climbing from a medical point of view. Swiss Med Wkly. 2012;142:w13688. https://doi.org/ 10.4414/smw.2012.13688.

10. Lutter C, El-Sheikh Y, Schöffl I, et al. Sport climbing: medical considerations for this new Olympic discipline. Br J Sports Med. 2017;51(1):2-3. https://doi.org/10.1136/ bjsports-2016-096871.

11. Ozimek M, Rokowski R, Draga P, et al. The role of physique, strength and endurance in the achievements of elite climbers. PLoS ONE. 2017;12(8):e182026. https://doi.org/ $10.1371 /$ journal.pone.0182026.

12. Woollings KY, McKay CD, Emery CA. Risk factors for injury in sport climbing and bouldering: a systematic review of the literature. Br J Sports Med. 2015;49(17):1094-9. https://doi. org/10.1136/bjsports-2014-094372.

13. Fryer S, Stone KJ, Sveen J, et al. Differences in forearm strength, endurance, and hemodynamic kinetics between maleboulderersandleadrockclimbers.EurJSportSci.2017; 1-7.https://doi.org/10.1080/17461391.2017.1353135.

14. Jones G, Johnson MI. A critical review of the incidence and risk factors for finger injuries in rock climbing. Curr Sports Med Rep. 2016;15(6):400-9. https://doi.org/10.1249/JSR. 0000000000000304.

15. Grzybowski C, Eils E. Therapeutisches Klettern - kaum erforscht und dennoch zunehmend eingesetzt (Therapeutic climbing - barely explored but widely used). Sportverletz Sportschaden. 2011;25(2):87-92. https://doi.org/10.1055/ s-0029-1245539.

16. Buechter RB, Fechtelpeter D. Climbing for preventing and treating health problems: a systematic review of randomized controlled trials. Ger Med Sci. 2011;9:Doc19. https:// doi.org/10.3205/000142.

17. Weber $H$. The hygienic and therapeutic aspects of climbing. Lancet. 1893;142(3661):1048-50. https://doi.org/10.1016/ S0140-6736(01)95058-5.

18. LazikD. Therapeutisches Klettern. Stuttgart:Thieme; 2008.

19. LeichtfriedV. Therapeutisches Klettern-eineExtremsportart geht neue Wege. In: Berghold F, Brugger H, Burtscher
M, et al., Hrsg. Alpin- und Höhenmedizin. Vienna: Springer Vienna;2015.S. 107-17.

20. Veser. Konzentriert durch Klettern - Therapeutisches Klettern bei Kindern mit ADHS. ergopraxis. 2009;2(4):18-21.

21. Engbert K, Weber M. The effects of therapeutic climbing in patients with chronic low back pain: a randomized controlled study. Spine (phila Pa 1976). 2011;36(11):842-9. https://doi.org/10.1097/BRS.0b013e3181e23cd1.

22. Mollenhauer A, Doll N, Renz P, et al. Therapeutisches Klettern in der Akutpsychiatrie. Pflegewissenschaft. 2011;11(09):453-61.

23. Wolf M, Mehl K. Experiential learning in psychotherapy: ropes course exposures as an adjunct to inpatient treatment. Clin Psychol Psychother.2011;18(1):60-74.https://doi.org/ $10.1002 /$ cpp.692.

24. Luttenberger K, Stelzer E, Forst S, et al. Indoor rockclimbing (bouldering) as a new treatment for depression: study design of a waitlist-controlled randomized group pilot study and the first results. BMC Psychiatry. 2015;15:201. https://doi.org/10.1186/s12888-015-0585-8.

25. Kleinstäuber M, Reuter M, Doll N, et al. Rock climbing and acute emotion regulation in patients with major depressive disorder in the context of a psychological inpatient treatment: a controlled pilot trial. Psychol Res Behav Manag. 2017;10:277-81. https://doi.org/10.2147/PRBM.S143830.

26. Mazzoni ER, Purves PL, Southward J, et al. Effect of indoor wall climbing on self-efficacy and self-perceptions of children with special needs. Adapt Phys Activ Q. 2009;26(3):259-73.

27. Schram Christensen M, Jensen T, Voigt CB, et al. To be active through indoor-climbing: an exploratory feasibility study in a group of children with cerebral palsy and typically developing children. Bmc Neurol. 2017;17(1):112. https:// doi.org/10.1186/s12883-017-0889-z.

28. Gallotta MC, Emerenziani GP, Monteiro MD, et al. Psychophysical benefits of rock-climbing activity. Percept MotSkills.2015;121(3):675-89.https://doi.org/10.2466/30. PMS.121c26x9.

29. Wallner S. Psychologisches Klettern Klettern als Mittel klinisch- und gesundheitspsychologischen Handelns. PsycholÖsterreich. 2010;5:396-404.

30. Kowald A, Zajetz AK, Hrsg. Therapeutisches Klettern: Anwendungsfelder in Psychotherapie und Pädagogik. 1. Aufl. Stuttgart: Schattauer; 2014.

31. Cunningham JA, Kypri K, McCambridge J. Exploratory randomized controlled trial evaluating the impact of a waiting list control design. Bmc Med Res Methodol. 2013;13:150. https://doi.org/10.1186/1471-2288-13-150.

32. Blumenthal JA, Babyak MA, Doraiswamy PM, et al. Exercise and pharmacotherapy in the treatment of major depressive disorder. Psychosom Med. 2007;69(7):587-96. https://doi. org/10.1097/PSY.0b013e318148c19a. 